\title{
PET/CT in Evaluating Pediatric Malignancies: A Clinician's Perspective
}

$\mathbf{T}$ with the functional imaging modality of PET has been an advancement in diagnostic imaging that has been welcomed by radiologists, nuclear medicine specialists, and oncologists. PET/CT has been used successfully in the diagnosis, staging, monitoring of response to therapy, and surveillance of various malignancies in adult patients $(1,2)$. However, the role of PET/ CT in the management of pediatric malignancies has not been well studied and, as a result, is less clearly defined in this population.

\section{See page 1923}

In this issue of The Journal of Nuclear Medicine, Tatsumi et al. report the use of PET/CT in the younger population in their retrospective study of $151 \mathrm{PET} / \mathrm{CT}$ examinations in 55 pediatric patients between 3 and $19 \mathrm{y}$ old (3). The results of this study are especially convincing in patients with Hodgkin disease (HD) as most of the PET/CT was performed in these patients. The utility of PET/CT in the management of HD makes sense, particularly in the nodular sclerosing variety, which is so common in adolescents. In these patients, therapy may

Received Sep. 5, 2007; revision accepted Sep. 11, 2007.

For correspondence or reprints contact: Noah Federman, MD, Mattel Children's Hospital at UCLA, David Geffen School of Medicine at UCLA, 10833 Le Conte Ave., Los Angeles, CA 90095.

E-mail: nfederman@mednet.ucla.edu COPYRIGHT (c) 2007 by the Society of Nuclear Medicine, Inc.

DOI: 10.2967/jnumed.107.046045 leave a patient with a fibrotic mass, which is still visible on CT or MRI. The ability of FDG PET to detect metabolic activity is very useful in differentiating between fibrotic scarring and residual active disease. These results have been corroborated in smaller studies of pediatric patients with $\mathrm{HD}$ and nonHodgkin lymphoma (NHL). Rhodes et al. showed that PET/CT had a higher sensitivity, compared with CT alone, in evaluating patients for recurrent disease (4). Miller et al. demonstrated improved accuracy of staging with PET/ CT in another small series of patients with lymphoma; there was a change in staging at the time of diagnosis in about one third of pediatric patients with HD and NHL (5).

There has been major improvement in the outlook for patients diagnosed with HD. The 5-y event-free survival (EFS) for children with favorableprognosis HD is $85 \%-100 \%$ (6). Patients with more advanced disease have a 5-y EFS in the range of 70\%-90\% (6). Thus, a major focus of attention for pediatric HD has shifted to the longterm toxicities of our treatment strategies. This has led to a "risk-stratified" approach to the use of radiation therapy, anthracyclines, and alkylating agents (6). In the current Children's Oncology Group trial, AHOD0031, using a riskadapted approach to modify therapy in pediatric patients with HD, PET/CT has been instituted for diagnosis, staging, and response to therapy (7).

The utility of PET/CT in the diagnosis, staging, evaluation of response to therapy, and long-term follow-up of other nonlymphoma pediatric malignancies has not been adequately studied. Tatsumi et al. report the use of $\mathrm{PET} / \mathrm{CT}$ in other pediatric malignancies in a group of 25 patients with bone and soft-tissue sarcomas, nasopharyngeal carcinomas, hepatoblastoma, ovarian cancer, and other malignancies (3). Although PET/CT may be helpful in the management of these various malignancies, the results of this study need to be repeated in larger trials that are histology specific.

The use of PET/CT can be helpful in the management of pediatric bone and soft-tissue sarcomas that demonstrate metabolic activity. It will assist in the identification of primary site, location of metastatic disease, and detection of recurrent disease. Thus, it will aid in the selection of therapeutic regimens and identify patients whose treatment needs adjustment. PET/CT has been particularly helpful in patients with inoperable sarcomas who have been treated with radiation for local control. This therapy may lead to scarring and fibrosis of bone and soft tissue that may be indistinguishable from persistent or recurrent disease without FDG PET to identify areas of hypermetabolic activity (8-10). Our experience has shown that the use of PET/CT in the management of Ewing sarcoma and osteosarcoma is useful in the initial staging period, response to therapy, and surveillance after chemotherapy in cases where 99m Tc-methylene diphosphonate bone scintigraphy is negative and FDG PET/ $\mathrm{CT}$ is positive on prior imaging. PET/CT may be useful in differentiating benign neurofibromas, usually metabolically inactive, from malignant peripheral nerve sheath tumors in patients with neurofibromatosis (11).

There has been much attention given to the long-term effects of the treatment of pediatric malignancies. These include development of cardiomyopathies, avascular necrosis of the hip, cognitive delay, 
early onset of heart disease, and increased risk of secondary malignancies. Children who receive alkylating chemotherapeutic agents and radiation as part of their treatment regimens-such as in patients with HD and sarcoma-are at particular risk for developing secondary malignancies. The increasing use of not only PET/CT but also serial $\mathrm{CT}$ and plain-film radiography in the management of pediatric malignancies raises the important consideration of radiation exposure in children. This exposure is not trivial in patients with HD and sarcoma, who often get CT scans for $5 \mathrm{y}$ as part of the surveillance for recurrent disease. It has been reported in numerous studies that children have an increased risk of developing secondary malignancies from radiation exposure compared with adults. In fact, by extrapolating data from atomic bomb survivors, this increased risk is by an order of magnitude greater than that of adults $(12,13)$. It appears that this risk may be related to the greater life span of children and, hence, longer latency period to develop cancer. It has also been hypothesized that children have a greater proportion of actively dividing cells making them inherently more susceptible to radiation-induced damage. Consequently, radiation exposure from PET/CT in children should adhere to the ALARA principle (as low as is reasonably achievable) to obtain diagnostic information (14).

The greatest contributor to overall radiation exposure in PET/CT is the whole-body diagnostic CT. In a study involving radiation exposure from whole-body FDG PET/CT in adults at 4 German institutions, the radiation dose from the diagnostic CT ranged from 14.1 to $18.6 \mathrm{mSv}$, the dose from CT topography ranged from 0.1 to 0.8 $\mathrm{mSv}$, and the dose from FDG PET ranged from 5.7 to $7.0 \mathrm{mSv}$ (15). The average total radiation dose of $25 \mathrm{mSv}$ from 1 adult whole-body PET/CT in this study is roughly equivalent to 125 chest radiographs (at an average 0.2 $\mathrm{mSv}$ per radiograph) and is equivalent to exposure of $7 \mathrm{y}$ of background radiation in an adult (16). In pediatrics, the radiation exposure from a standard
PET/CT with diagnostic contrastenhanced CT and CT topography for a 55-kg 15-y-old, a 20-kg 5-y-old, and a $10-\mathrm{kg} 1-\mathrm{y}$-old is 21,18 , and $15 \mathrm{mSv}$, respectively. Using combined FDG PET/low-dose CT, the radiation exposure is reduced to $9 \mathrm{mSv}$ in the $55-\mathrm{kg}$ adolescent, $8 \mathrm{mSv}$ in the $20-\mathrm{kg}$ child, and $7 \mathrm{mSv}$ in the $10-\mathrm{kg}$ baby (17). However, it is not always possible or recommended to use low-dose CT for image attenuation in place of diagnostic spiral chest CT. This is particularly important in the staging of sarcoma patients, where FDG PET is less sensitive than spiral CT for the detection of pulmonary metastases (18).

From a clinical perspective, it is also important to keep in mind the feasibility of the PET/CT study in children. Young children may require anesthesia to keep still for the duration of the scan. The use of anesthesia in pediatrics is not without risk. A retrospective review of 16,467 pediatric sedations performed between 1997 and 2003 showed a $0.4 \%$ incidence of adverse respiratory events (19). In general, most children above the age of $5 \mathrm{y}$ are able to undergo PET/CT examinations without the use of anesthesia. Franzius and Schober have reported that only $30 \%$ of 355 pediatric patients younger than $3 \frac{1}{2} \mathrm{y}$ required sedation to perform the PET/ CT (20).

In summary, combined FDG PET/ CT is a modality that may improve the evaluation and surveillance of pediatric malignancies. This has been shown convincingly by Tatsumi et al. (3). In HD and pediatric sarcomas, PET/CT appears to be an important tool in the management of these diseases. However, the use of PET/CT in other pediatric malignancies has yet to be rigorously studied. Furthermore, the timing, interval, and number of needed PET/CT scans in HD and sarcoma has not been clearly shown. Ongoing research is necessary to refine the ALARA principle in the use of PET/CT without sacrificing diagnostic information. Combined PET/MRI may be an option to reduce radiation exposure in the future. However, this may not be feasible because CT is superior to MRI in evaluation of lung parenchyma for metastatic disease, and children may require sedation for the extended duration of an MRI.

Noah Federman
Stephen A. Feig
Mattel Children's Hospital
David Geffen School of Medicine at UCLA
Los Angeles, California

\section{REFERENCES}

1. Czernin J, Allen-Auerbach M, Schelbert HR. Improvements in cancer staging with PET/CT: literature-based evidence as of September 2006. J Nucl Med. 2007;48(suppl):78S-88S.

2. Czernin J, Schelbert HR. PET/CT imaging: facts, opinions, hopes, and questions. J Nucl Med. 2004; 45(suppl): $1 \mathrm{~S}-3 \mathrm{~S}$.

3. Tatsumi M, Miller JH, Wahl RL. ${ }^{18}$ F-FDG PET/ $\mathrm{CT}$ in evaluating non-CNS pediatric malignancies. J Nucl Med. 2007;48:1923-1931.

4. Rhodes MM, Delbeke D, Whitlock JA, et al. Utility of FDG-PET/CT in follow-up of children treated for Hodgkin and non-Hodgkin lymphoma. J Pediatr Hematol Oncol. 2006;28: 300-306.

5. Miller E, Meltser U, Avrahami G, et al. Role of ${ }^{18} \mathrm{~F}$-FDG PET/CT in staging and follow-up of lymphoma in pediatric and young adult patients. J Comput Assist Tomogr. 2006;30:689694.

6. Hudson MM, Donaldson SS. Treatment of pediatric Hodgkin's lymphoma. Semin Hematol. 1999;36: 313-323.

7. NCT00025259. National Institutes of Health Children's Oncology Group AHOD0031. Available at: www.cancer.gov/clinicaltrials/COG-AHOD0031. Accessed October 19, 2007.

8. Arush MW, Israel O, Postovsky S, et al. Positron emission tomography/computed tomography with 18-fluoro-deoxyglucose in the detection of local recurrence and distant metastases of pediatric sarcoma. Pediatr Blood Cancer. January 24, 2007 [Epub ahead of print].

9. Iagaru A, Quon A, McDougall IR. F-18 FDG-PET/ CT evaluation in osseous and soft tissue sarcomas. Clin Nucl Med. 2006;31:754-760.

10. McCarville MB, Christie R, Daw NC. PET/CT in the evaluation of childhood sarcomas. AJR. 2005; 184:1293-1304.

11. Otsuka H, Graham MM, Kubo A, Nishitani H. FDG-PET/CT findings of sarcomatous transformation in neurofibromatosis: a case report. Ann Nucl Med. 2005;19:55-58.

12. Pierce DA, Shimizu Y, Preston DL, et al. Studies of the mortality of atomic bomb survivors: Report 12, Part 1-Cancer: 1950-1990. Radiat Res. 1996; 146:1-27.

13. Brenner DJ, Elliston CD, Hall EJ, et al. Estimating cancer risks from pediatric CT: going from the qualitative to the quantitative. Pediatr Radiol. 2002; 32:228-231.

14. Slovis TL. Conference on the ALARA (as low as reasonably achievable) concept in pediatric CT: intelligent dose reduction. Pediatr Radiol. 2002;32:217-218. 
15. Brix G, Lechel U, Glatting G. Radiation exposure of patients undergoing whole-body dual-modality ${ }^{18}$ F-FDG PET/CT examinations. J Nucl Med. 2005; 46:608-613.

16. Frush DP, Donnelly LF, Rosen NS. Computed tomography and radiation risks: what pediatric health care providers should know. Pediatrics. 2003;112:951-957.
17. Byrne A, Nadel H. Whole body low dose ${ }^{18} \mathrm{~F}-\mathrm{FDG}$ $\mathrm{PET} / \mathrm{CT}$ in pediatric oncology [abstract]. $\mathrm{J} \mathrm{Nucl}$ Med. 2007;48(suppl 2):118P.

18. Franzius C, Daldrup-Link HE, Sciuk J, et al. FDGPET for the detection of pulmonary metastases from malignant primary bone tumors: comparison with spiral CT. Ann Oncol. 2001;12:479-486.
19. Sanborn PA, Michna E, Zurakowski D, et al. Adverse cardiovascular and respiratory events during sedation of pediatric patients for imaging examinations. Radiology. 2005;237:288-294.

20. Franzius C, Schober O. Is PET/CT necessary in paediatric oncology? Eur J Nucl Med Mol Imaging. 2006;33:960-965. 